\title{
Padrões de comportamento de busca e uso de informação por pesquisadores de biologia molecular e biotecnologia*
}

\author{
Isabel Merlo Crespo \\ Mestre em comunicação e informação pela Universidade Federal do \\ Rio Grande do Sul. \\ E-mail: icrespo@pucrs.br
}

\section{Sônia Elisa Caregnato \\ Doutora em ciência da informação pela Sheffield University. E-mail: sonia.caregnato@ufrgs.br}

\section{Resumo}

O comportamento de busca e uso de informação dos pesquisadores da área de biologia molecular e biotecnologia foi analisado com base no modelo de David Ellis, verificando como a informação científica em meio digital altera este comportamento. Constituiu-se de pesquisa qualitativa, que, para a coleta de dados, utilizou questões abertas, com entrevista aos pesquisadores do Departamento de Biologia Molecular e Biotecnologia da Universidade Federal do Rio Grande do Sul. Os dados foram trabalhados em uma base de dados desenvolvida no software de análise qualitativa Nvivo versão 2.0, em conformidade com técnicas de codificação o método comparativo constante. Os resultados mostraram um perfil de comportamento de busca e uso de informação do grupo de pesquisadores, com suas principais características, em vários aspectos, geradas pelo uso das tecnologias, destacando a nãolinearidade da busca e obtenção da informação. Constatou-se que os periódicos científicos eletrônicos são a principal fonte de informação destes pesquisadores, e que são amplamente utilizados e aceitos, mas o mesmo não acontece com aqueles de acesso livre, ainda usados de modo restrito. Conclui, também, que o modelo de Ellis é válido, com ampliações e modificações. Sugere novos temas de estudo relacionados ao enfoque pesquisado.

\section{Palavras-chave}

Busca e uso de informação. Modelos de comportamento de busca de informação. Comunicação científica. Biologia molecular e biotecnologia.

\section{Behavior standards of information search and use referring to molecular biology and biotechnology}

\section{Abstract \\ The information seeking and use behavior of Molecular Biology and Biotechnology researchers has been analyzed based on David Ellis ' model, in order to verify how scientific information published in digital media affects this behavior. A qualitative research approach was used and data was collected by means of an interview guide with a set of open questions, applied to Faculty at the Molecular Biology and Biotechnology Department of the Rio Grande do Sul Federal University (UFRGS). The data was managed as a project on the Nvivo 2.0 software for qualitative analysis, employing codification techniques, as well as the constant comparative method. The final results have shown a pattern in the information seeking and use behavior among the group of researchers characterized, in many aspects, by the use of technology, such as the use of non-linear information seeking strategies. It has been noted that electronic scientific journals are the most important source of information amongst these researchers, and that they are widely used and accepted. However, the same does not apply to those of free access, which are still used in a restricted way. It has also been concluded that, the Ellis is a valid model, nevertheless, under some improvements and changes. This paper suggests new study topics related to the subject studied.}

Keywords

Information seeking and use. Information seeking behavior models. Scientific communication. Molecular biology and biotechnology.

\section{INTRODUÇÃO}

A biologia molecular e a biotecnologia são áreas que se caracterizam como produtoras e consumidoras de conhecimento científico e tecnológico atual, necessitando, assim, de fontes de informações bastante recentes e completas para fundamentar suas pesquisas.

Em decorrência disso, os pesquisadores dessas áreas utilizam amplamente os periódicos e as publicações científicas eletrônicas, por serem muito atualizados, de fácil acesso e confiáveis.

Esse direcionamento de suas práticas de comunicação para o uso intenso dos periódicos é característico das áreas científicas duras, o que as diferencia das ciências humanas e sociais, nas quais o livro ocupa posição central. Meadows (1999) demonstra essa situação a partir das diferentes práticas de citações adotadas pelos pesquisadores, explicando que $82 \%$ das citações dos trabalhos dos cientistas das áreas chamadas duras eram oriundas dos periódicos, enquanto para as ciências sociais o percentual era de somente 29\%. Quanto aos livros, a situação era oposta, sendo que, nas ciências duras, essa fonte era utilizada em $12 \%$ dos trabalhos e, nas ciências, sociais era usada em $46 \%$ das referências.

Outra característica que pode ser verificada no que se refere às práticas de comunicação dos pesquisadores é quanto ao número de autores por artigo, o que reflete o tipo de organização em equipes, adotado em algumas áreas. Tal situação pode ser observada na comparação apresentada por Meadows (1999) entre a bioquímica, a sociologia, a psicologia e a economia. Entre as áreas descritas, a bioquímica era a que possuía o maior índice de artigos, com quatro ou mais autores, e era a que tinha o menor percentual de artigos com um só autor. Outra questão ressaltada por Meadows (1999) é referente ao tamanho das publicações. Nas ciências sociais, ele é maior do que nas áreas duras, nas quais os principais meios de

\footnotetext{
* Extraído da dissertação de mestrado defendida no Programa de PósGraduação em Comunicação e Informação (PPGCOM) da Universidade Federal do Rio Grande do Sul, em 2005, com a orientação da professora doutora Sônia Elisa Caregnato.
} 
publicação são os periódicos científicos e os anais de eventos.

A preferência por fontes eletrônicas pode ser averiguada também mediante a quantidade de fontes de informação em meios eletrônicos disponíveis para essas áreas, como as bases de dados, e do elevado número de periódicos destes enfoques disponibilizados no Portal de Periódicos da Capes. Ali verificou-se que somente para a biologia existem 231 títulos, sem observar as demais áreas relacionadas e que também são fonte de pesquisa.

O uso predominante de periódicos científicos por estas áreas é confirmado também por Grefsheim, Franklin e Cunningham (1991), em estudo que desenvolveram para reconhecer as necessidades de informação de pesquisadores da área de biotecnologia. Nele destacam que o livro não era um recurso muito utilizado, devido ao crescimento rápido do campo e à demora na publicação deste tipo de documento, tornando-o uma fonte de menor utilização, principalmente se comparada ao artigo de periódico. Também foi averiguado que é por meio dos periódicos científicos que os pesquisadores obtêm as informações que consideram de maior utilidade para as suas atividades de pesquisa.

Atualmente, as publicações científicas periódicas adotaram a Internet como um suporte para disponibilizar seus conteúdos. Inicialmente, no entanto, esse novo tipo de publicação tinha pouca aceitação no meio científico, e era acessível a um público restrito. Com o passar do tempo e devido a vários fatores, como o barateamento dos custos, as tecnologias ficaram disponíveis para um número maior de pessoas e com melhor qualidade. A aceitação e o uso desse recurso se ampliou, e ele passou a ser adotado, embora de modo diferenciado, em várias áreas da ciência.

Surgimento dos periódicos científicos eletrônicos ampliou as necessidades de estudos sobre as práticas de comunicação dos diferentes grupos de pesquisadores, pois eles têm potencial para gerar novas formas de busca e utilização de informações pelos indivíduos. Esses novos tipos de comportamentos podem ser verificados em estudos de busca e uso de informação, que procuram conhecer a estrutura, bem como as características que envolvem a interação do sujeito com os recursos, serviços e fontes de informação.

Otrabalho aqui relatado constituiu-se de um estudo, à luz do modelo de David Ellis, sobre o comportamento de busca e uso de informação de pesquisadores das áreas de biologia molecular e biotecnologia da Universidade Federal do Rio Grande do Sul (UFRGS) para o desenvolvimento de suas atividades de pesquisa e docência. Verificou-se como realizam a busca e uso de informação e de que modo essas atividades foram alteradas com a introdução das tecnologias digitais em rede, principalmente com o periódico científico eletrônico.

\section{COMPORTAMENTO DE BUSCA E USO DE INFORMAÇÃO}

O comportamento de busca de informação resulta do reconhecimento de alguma necessidade, ou seja, o indivíduo verifica que a informação que possui não corresponde ao que precisa e, então, age para satisfazer tal necessidade (WILSON, 1981). Krikelas (1983, p. 6-7, tradução nossa) o define deste modo:

[...] todas as atividades de um indivíduo que sejam realizadas para identificar mensagens que satisfaçam às necessidades percebidas. Ou seja, busca de informação começa quando alguém percebe que o conhecimento atual possuído é menor que o desejado para tratar de algum assunto.

Verifica-se que o comportamento de busca de informação é direcionado para as ações realizadas pelos indivíduos visando a localizar o que procuram. Para tanto, requerse a execução de várias atividades. Para Marchionini (1998), a busca de informação é composta por diversos estágios que compõem um processo, os quais envolvem aspectos relacionados aos sentimentos que motivam os indivíduos a sua percepção a respeito da questão que precisam pesquisar, entre outros. Os estágios apresentados pelo autor são os seguintes:

a) reconhecimento e aceitação do problema de informação;

b) definição e entendimento do problema;

c) seleção das fontes de informação;

d) formulação da pergunta;

e) execução da pesquisa;

f) verificação dos resultados;

g) extração da informação.

Vários fatores podem interferir no comportamento de busca e uso de informação, auxiliando ou não o indivíduo a suprir suas necessidades informacionais. Entre esses aspectos estão os que Wilson (1981) define como barreiras, que são as pessoais, as interpessoais e as do 
ambiente no qual o indivíduo está inserido. Essas barreiras mostram que o atendimento das necessidades de informação não depende somente da execução da busca, pois a interferência desses outros fatores pode acarretar que as mesmas não sejam satisfeitas ou que sejam realizadas após longo período.

O comportamento de busca e uso de informação de cientistas e acadêmicos já é foco de estudo da ciência da informação há algum tempo, direcionando-se para a análise de grupos específicos. Em 1948, na Conferência de Informação Científica da Royal Society, já era possível identificar trabalhos publicados sobre o assunto (WILSON, 1999).

Seguiram-se diversos trabalhos com enfoques variados, voltados principalmente para estudos sobre cientistas sociais. No início da década de 1970 , os pesquisadores buscaram dar às suas pesquisas um enfoque qualitativo, preocupando-se em analisar o comportamento humano, direcionando, dessa forma, vários estudos posteriores.

Atualmente, os estudos de comportamento estão voltados para a aplicação de métodos qualitativos, seguindo a linha dos trabalhos desenvolvidos nessa área no Reino Unido, que acabaram por criar uma tradição na investigação do comportamento humano, como nos estudos de T. D. Wilson, David Ellis, entre outros. Essa tendência também pode ser verificada nas pesquisas realizadas entre autores americanos, como Dervin e Nilan (1986) e Kuhlthau (1991), que buscaram identificar os sentimentos e motivações do indivíduo no processo de busca e uso de informação.

Entre os estudos basilares está o de Wilson (1981), que pesquisou o comportamento de busca de informação de pesquisadores e cientistas e procurou criar um modelo para diferenciar os conceitos de necessidade e busca de informação. Após, recebendo a influência das pesquisas de Ellis (1989a,1989b) e Ellis, Cox e Hall (1993), Wilson alterou sucessivas vezes o seu primeiro modelo de comportamento informacional e apresentou uma nova versão (WILSON, 1997). Posteriormente, Wilson (1999) também comparou e relacionou diversos modelos, criando o conceito de estrutura complexa, que abrange o comportamento informacional, identificada como a estrutura mais ampla na qual se insere o comportamento de busca de informação e, nesta, o comportamento de busca em sistemas de informação.

Brenda Dervin, outra estudiosa da área, criou a metodologia sense-making, a partir de 1972, e a sistematizou a partir de 1983, baseando-se na teoria da comunicação. A metodologia sense-making direciona-se para o estudo do indivíduo, verificando como se comunica, percebe e sente o contato com mídia, instituições etc. (DERVIN; NILAN, 1986).

Outro modelo a ser destacado é o desenvolvido por Kuhlthau (1991), que estudou os padrões do processo de busca e uso de informação de estudantes de graduação, durante a realização de seu trabalho de conclusão do curso. A autora definiu um modelo centrado no indivíduo, levando em consideração os sentimentos, pensamentos e ações que ocorrem durante o processo. Esse modelo foi chamado de Information Search Process (ISP), o qual é formado por um conjunto de seis etapas seqüenciais: início, seleção, exploração, formulação, coleta e apresentação.

Para este trabalho, teve fundamental importância o estudo desenvolvido por David Ellis, que criou um modelo de comportamento de busca de informação, em seu trabalho de doutoramento apresentado em $1987^{*}$ na Universidade de Sheffield. O modelo descrito é centrado em aspectos cognitivos da busca de informação e é formado por padrões de comportamento que não se caracterizam como fases de um processo seqüencial. Ellis fundamentou-se no estudo do comportamento de grupos de cientistas sociais de vários departamentos da Universidade de Sheffield e, por meio dele, fez recomendações para o design de sistemas de recuperação da informação (ELLIS, 1989a, 1989b). Esse modelo foi estruturado em seis categorias, que não estão organizadas em ordem seqüencial, podendo sobrepor-se (ELLIS, 1989a, 1989b). São elas:

a) iniciar: é composta pelas atividades efetuadas no começo da busca de informação e que trazem informações que podem basear posteriores ampliações da busca;

b) encadear: abrange as buscas de informação nas quais os indivíduos efetuam ligações entre as citações. Essas relações podem permitir a localização de outros materiais relevantes e, assim, realizar formas de conexão entre o que foi localizado e as novas informações;

c) navegar: caracteriza-se como um modo de pesquisa não muito objetiva, ou seja, é uma busca semidirecionada, ou semi-estruturada, a uma área de interesse amplo;

\footnotetext{
* ELLIS, David. The derivation of a behavioural model for information retrieval system design. Thesis (Ph.D.) - Department of Information Studies, University of Sheffield, Sheffield, 1987.
} 
d) diferenciar: abrange as atividades efetuadas na avaliação das diferenças entre as fontes como um filtro para analisar o material identificado;

e) monitorar: compreende o monitoramento das fontes de informação específicas;

f) extrair: são as atividades sistemáticas efetuadas pelo usuário em uma fonte específica para obter o material de que necessita.

Este modelo inicial de comportamento de busca de informação de Ellis foi posteriormente ampliado por Ellis, Cox e Hall (1993), a partir de um estudo comparativo entre cientistas sociais e cientistas de áreas exatas (químicos e físicos), passando de seis para oito categorias. As duas novas categorias são as seguintes:

Verificar: atividades vinculadas com a verificação da acuracidade da informação. A categoria não foi destacada no modelo inicial de Ellis como uma característica específica, apesar de terem sido observados comportamentos similares no estudo com cientistas sociais. Várias situações que ressaltam esse comportamento foram reconhecidas; por exemplo, a maioria dos químicos descreveu que estava ciente da possibilidade de ocorrência de erros, especialmente os tipográficos (ELLIS; COX; HALL, 1993).

Finalizar: atividades da busca de informação ao final de um tópico ou projeto, por exemplo, durante a preparação de artigos para publicação. Essa categoria também não foi identificada no modelo inicial de Ellis (1989a, 1989b) como merecedora de destaque em separado, embora também tenha sido mencionada pelos cientistas sociais. Na análise de Ellis, Cox e Hall (1993), ela foi verificada em várias etapas das atividades realizadas pelos cientistas, descrita na fase de coleta ao início ou no decorrer do projeto.

O modelo ampliado por Ellis, Cox e Hall (1993) é relevante, pois foi estruturado em características de comportamento amplas e que se adequam a várias áreas do conhecimento, baseando-se em detalhada pesquisa empírica e influenciando diversos trabalhos. Ressaltamse, dentre esses, os de Choo, Detlor e Turnbull (1998, 2000), em que o modelo inicial de seis categorias foi utilizado para um estudo de comportamento de busca de informação na web, com 34 usuários, em sua maioria especialistas em tecnologia, gerentes e equipes de pesquisa, marketing e consultoria que utilizam a web em suas atividades diárias. Também é importante o de Meho e Tibbo (2003), que desenvolveram uma pesquisa com professores das áreas de ciências sociais, a qual confirmou o modelo de Ellis, de seis categorias, e organizaram uma estrutura em estágios, além de incluir outras funções.

\section{PROCEDIMENTOS METODOLÓGICOS}

estudo adotou um enfoque qualitativo partindo da estrutura do modelo de comportamento de busca e uso de informação de David Ellis.

Os sujeitos do estudo foram os docentes/pesquisadores que integram o Departamento de Biologia Molecular e Biotecnologia da Universidade Federal do Rio Grande do Sul (UFRGS), que possuem doutorado e/ou pósdoutorado, são responsáveis por disciplinas em cursos de graduação em ciências biológicas, medicina e outros e também atuam como orientadores em programas de pósgraduação. A escolha do grupo de pesquisadores da área de biologia molecular e biotecnologia, para o estudo, deuse por diversos fatores. Primeiramente pelo uso intensivo que eles fazem de periódicos científicos, especialmente em meio eletrônico, caracterizando-se como sua principal fonte de informação e como um importante canal para disseminação de suas pesquisas. Também, pela verificação de avanços rápidos nessa área da ciência, o que contribui para demonstrar a importância da atualização constante do cientista e, conseqüen-temente, da busca de informações que embasem a construção do conhecimento. Finalmente, a escolha deu-se pela existência de quantidade significativa de fontes de informação em meio eletrônico nessa área, como bases de dados e periódicos eletrônicos, o que mostra a importância que esse tipo de fonte possui para o campo de estudo.

Para a coleta de dados foram aplicadas entrevistas semiestruturadas, com questões abertas, partindo da mais abrangente para a mais específica, adotando o formato de um guia de entrevista. Esse tipo de entrevista possui suas bases metodológicas na pesquisa qualitativa e, segundo Flick (2004), permite ao entrevistador que ele decida quando e em qual ordem irá realizar as perguntas definidas. As questões aplicadas foram formuladas a partir do modelo de busca de informação desenvolvido por David Ellis, em seu estudo do ano 1989b, ampliado em 1993. Além disso, foram incorporadas questões adicionais sobre busca e uso de informações científicas em recursos eletrônicos on-line, especialmente os periódicos eletrônicos, fonte de informação pouco utilizada no período em que Ellis desenvolveu seu modelo. Foi realizado um pré-teste, aplicado a três 
pesquisadores da área biomédica, com o objetivo de avaliar as perguntas formuladas, permitindo assim a sua melhor adequação.

Após, foram efetuadas as entrevistas com o grupo selecionado, gravadas e posteriormente transcritas. As entrevistas foram inseridas no programa de análise qualitativa, Nvivo 2.0. Esse programa se enquadra na categoria de CAQDAS (Computer Assisted Qualitative Data Analysis Software) que visa à organização das informações em níveis e subníveis e permite diversas formas de tratamento e recuperação das informações (TEIXEIRA; BECKER, 2001). O Nvivo 2.0 foi utilizado, considerando-se a sua capacidade de realizar todas as verificações e estruturações necessárias para a checagem detalhada dos dados.

Para a análise dos dados, realizou-se uma verificação detalhada de cada linha da resposta e identificaram-se os rótulos conceituais, ou seja, foi feita a codificação das informações, que foram inseridas nas oito categorias do modelo de comportamento de busca e uso de informação desenvolvido por David Ellis.

Também foram criadas outras classificações para os conceitos que não se enquadraram nas categorias já existentes. Para verificar em que categoria cada rótulo conceitual se encaixara, foi utilizado o método comparativo constante (STRAUSS; CORBIN, 1990), que consiste na identificação de similaridades e diferenças entre os conceitos. Durante a análise dos dados, percebeu-se a necessidade da criação de outras estruturas de organização dos dados, além dos predefinidos.

A partir da análise dos dados levantados, foram apresentados os resultados obtidos.

\section{ANÁLISE E RESULTADOS}

Por meio do exame das categorias do modelo de Ellis e da comparação com a literatura sobre o assunto, foi possível ampliá-lo e modificá-lo. Uma nova categoria, denominada "personalizar", foi inserida, direcionandose para aspectos não contemplados no modelo. As oito categorias do modelo de Ellis - iniciar, encadear, navegar, diferenciar, monitorar, extrair, verificar, finalizar - foram confirmadas, mas adquiriram enfoques ampliados, principalmente pela utilização das novas tecnologias.

\section{Iniciar}

Nesta investigação, esse padrão de comportamento foi verificado em todas as entrevistas, nas quais se pode identificar uma forma de comportamento típico do início das pesquisas, que é o de buscar os assuntos em bases de dados on-line disponíveis na Internet, principalmente no Web of Science e na Pubmed, sem procurar em documentos impressos, como era caracterizado anteriormente.

Identificou-se que, mediante as bases de dados on-line, informando os assuntos a serem localizados, pesquisadores obtêm as referências iniciais de seus trabalhos e após acessam e baixam os artigos que lhes interessam.

Contudo, cumpre destacar que, para alguns dos entrevistados, este comportamento característico do início repete-se durante várias fases da busca de informação, já que para eles esse processo não possui uma finalização e necessita ser constantemente refeito.

Reconheceu-se que essa categoria não foi verificada em atividades de busca em catálogos de bibliotecas, como apresentado por Ellis (1989a). Também foi identificada nas buscas informais: através do contato com outros colegas do mesmo departamento, de outros departamentos da universidade ou até de diferentes laboratórios e universidades. Outra atividade peculiar foi a de analisar o que está sendo desenvolvido em outros laboratórios e instituições de pesquisa. Para tanto, os pesquisadores utilizam como ferramentas os diretórios de busca, que permitem uma varredura.

Como comportamento padrão nesta categoria foi possível identificar a busca de referências em fontes de informação em meio eletrônico, que o pesquisador já selecionou previamente como as mais adequadas. Dessa forma ele obtém as informações iniciais para embasar o trabalho que está realizando ou que irá desenvolver. Essa busca pode ser diferenciada em função do conhecimento que o pesquisador tem do assunto que está buscando e do domínio dos recursos de busca de informação.

\section{Encadear}

$\mathrm{Na}$ análise das entrevistas, foram identificadas instâncias específicas da categoria "encadear", na realização de atividades de encadeamento para frente no tempo (forward chaining) e encadeamento para trás no tempo (backward chaining). No estudo de Ellis (1989a, 1989b), o encadeamento para trás no tempo era visto como uma forma tradicional de busca de informação e considerada de grande importância e bastante usual nos estudos com cientistas sociais. Já o encadeamento para a frente era reconhecido como uma exceção, uma inovação, devido à necessidade de uso de recursos específicos para recuperar 
esse tipo de informação. Ressalta-se, neste estudo, que, com os recursos eletrônicos, as diferenças entre esses dois aspectos se estreitaram e ambos ficaram muito mais fáceis de serem realizados. Por meio de ferramentas, como bases de dados de citações, é possível verificar todas as referências bibliográficas de determinado artigo e também as referências em que determinado autor foi citado, estabelecendo as mais variadas conexões entre as referências.

Neste estudo, reconheceu-se que essa categoria foi alterada pelo comportamento corrente entre pesquisadores entrevistados, possibilitando ampliação da definição original de Ellis. Neste trabalho, entende-se a categoria "encadear" como as ligações que os indivíduos efetuam entre os documentos por meio de hiperlinks a citações, a textos na íntegra, a capítulos de livros, a sites de instituições, entre outros. Isso se deve às tecnologias que modificaram a forma de acesso à informação, aumentando as opções de busca e localização de informação e a relação entre as fontes de informação e os documentos.

\section{Navegar}

Observou-se que os pesquisadores utilizam diversos tipos de fontes para realizar as atividades desta categoria, recorrendo a bases de dados, mecanismos de busca na Internet e a periódicos eletrônicos, disponíveis on-line pela Internet. Esta categoria foi reconhecida principalmente pelo uso de ferramentas de busca disponíveis na Internet, como o Google e o Yahoo, pelos quais são localizados, mediante palavras consideradas relevantes para o pesquisador, os mais diversos tipos de materiais, desde artigos científicos a sites de instituições ou pessoais.

Outro ponto a ser destacado em relação a esta categoria foi o reconhecimento do uso desse tipo de pesquisa mais ampla, dentro de periódicos científicos eletrônicos online da área de interesse e de áreas diretamente relacionadas. Os pesquisadores verificam o conteúdo dos periódicos, com o auxílio de ferramentas de busca gerais disponibilizadas pelo próprio site da revista, ou examinando superficialmente o sumário de diversos fascículos, e procuram identificar tópicos de seu interesse.

Deve-se ressaltar que atualmente há uma utilização do conceito de "navegar" (browsing) diferente do inicial adotado por Ellis. Ele o empregava no sentido de uma forma de pesquisa mais superficial, sem que fosse imprescindível a realização de ligações entre as informações. No ambiente proporcionado pela Internet, a situação se modifica, e o termo é aplicado amplamente para toda a ação de mover-se de uma página a outra, por meio de links hipertextuais da web.

\section{Diferenciar}

Neste estudo, a categoria diferenciar foi identificada em vários momentos, principalmente nas atividades em que o pesquisador adota o critério da relevância do assunto abordado no artigo, focando somente nos documentos que contenham informações sobre seu tópico ou diretamente relacionadas a ele.

Verifica-se, também, que esta categoria pode ser influenciada pelo objetivo que o pesquisador pretende alcançar com o levantamento de informações, pois depende do tipo de trabalho que necessita fazer.

Observou-se como um fator que contribui para a seleção, a abrangência do artigo, ou seja, se para aquela área específica a informação é relevante, adota-se determinado tipo de metodologia ou outro.

Destaca-se, ainda, que são particularidades da categoria a diferenciação baseada na qualidade do periódico, pois os pesquisadores direcionam-se para os que são reconhecidos em sua área, e também a diferenciação baseada na confiança na autoria do documento, a qual foi identificada como um fator que transfere valor ao artigo no estudo de Ellis, Cox e Hall (1993).

\section{Monitorar}

Neste estudo, reconheceu-se, principalmente, a categoria "monitorar" aplicada à verificação de periódicos científicos. Isso também é observado no estudo de Ellis (1989a), no qual os cientistas sociais se utilizavam de fontes que permitissem acompanhar vários periódicos selecionados.

Identifica-se a categoria quando o pesquisador elege revistas científicas eletrônicas de sua área de interesse, as quais são periodicamente verificadas, por meio de algum recurso específico, como, por exemplo, pelo acesso à homepage do mesmo.

Outra questão observada foi a ampliação do leque de atividades abrangidas pela categoria, graças ao uso generalizado de recursos eletrônicos. Como exemplo, destaca-se o uso de avisos automáticos que são enviados para o e-mail de quem se cadastrar nesses serviços. Esses avisos são encaminhados periodicamente, dependendo 
da publicação de novos fascículos dos periódicos, ou quando for disponibilizada alguma notícia sobre as áreas de interesse em que o usuário havia se cadastrado.

A possibilidade de se cadastrar nesses serviços de alerta permite que as atividades sejam simplificadas, pois o indivíduo não necessita mais acessar muitas fontes para estar informado em sua área de interesse. Ele recebe sistematicamente materiais referentes aos tópicos desejados.

\section{Extrair}

Esta categoria foi identificada em todas as entrevistas e em vários momentos delas, nas atividades de busca de informação em bases de dados específicas, ou diretamente em periódicos, teses, dissertações e outros.

Também foi reconhecida a busca e uso de informação desse grupo de pesquisadores em bases de dados on-line, principalmente o Web of Science e Pubmed, e em ferramentas de acesso ao conteúdo geral. Percebe-se que as bases de dados são consideradas uma fonte essencial para áreas como as biomédicas e outras relacionadas a elas, que dispõem de diversos recursos, muito atualizados, e possuem várias ferramentas de busca e localização de informação.

Outro ponto identificado em relação à busca de informação foi o domínio que o grupo de pesquisadores possui das ferramentas de busca e recursos para refinálas, ou seja, saber como restringi-las, recuperando informações relevantes, e não grande quantidade de informação, o que dificultaria a análise e a seleção. Segundo Meadows (1999), a recuperação de muita informação em buscas eletrônicas se deve ao fato de que os sistemas podem gerar sobrecarga de informações, recuperadas por vários pontos de acesso, quando o usuário não domina os recursos para direcioná-las e torná-las mais objetivas.

O comportamento-padrão desta categoria foi reconhecido como a busca sistemática, realizada, principalmente, em bases de dados específicas, disponíveis na Internet, identificando os materiais de seu interesse.

\section{Verificar}

Neste estudo, foi possível identificar a ocorrência desta categoria, mas de um modo diferenciado do de Ellis, pois os cientistas não se preocupavam com a checagem das informações para verificar a qualidade dos dados contidos no artigo. Para eles, o verificar se dá pela possibilidade de repetir em laboratório os experimentos apresentados nos artigos, preocupando-se, sim, com a garantia da veracidade das conclusões e análises alcançadas, e não com possíveis erros de grafia ou mesmo de digitação.

Aparentemente os pesquisadores não costumam checar as informações contidas nos artigos, por acreditarem que os erros e/ou fraudes acontecem com baixíssima freqüência, já que as conseqüências que sofreriam os cientistas, caso publicassem resultados adulterados, são suficientemente pesadas para inibir esse tipo de iniciativa. Outro fator que colabora para a confiança dos pesquisadores no conteúdo divulgado nas fontes de informação é o procedimento de "revisão por pares". Os referees analisam os artigos que são submetidos à publicação, verificando se correspondem a padrões requeridos e assegurando que sejam aceitos somente os artigos que tenham qualidade científica (ZIMAN, 1979). Esse processo é visto pela comunidade científica como uma garantia da qualidade, considerada, assim, como um fator que libera o pesquisador de verificações nos documentos, porque já foram submetidos a avaliações.

Verificou-se que os cientistas buscam informações em fontes que consideram confiáveis, nas quais é disponibilizado material que já possua algum crivo científico, como nas bases de dados que indexam periódicos com corpo editorial, corpo de revisores e fator de impacto.

\section{Finalizar}

Nesta pesquisa, quando foi indagado aos pesquisadores se identificavam o momento final do seu processo de busca de informação, todos responderam que é difícil caracterizar o final, pois consideram que a busca de informação deve estar sempre ocorrendo e que não se caracteriza como uma atividade que acontece somente em determinadas fases de uma pesquisa, mas que permeia todo o processo. Segundo Coelho et al. (1989), o comportamento de busca e uso de informação de pesquisadores é direcionado para a busca constante de informação durante todas as etapas de desenvolvimento de um projeto, e não somente em uma fase específica.

Nesse sentido, verificou-se que os pesquisadores efetuavam suas buscas durante todas as etapas de desenvolvimento de uma pesquisa, muitas vezes, finalizando suas buscas por informação somente próximo da data de envio do resultado de seu trabalho, como, por exemplo, no prazo de um artigo a ser submetido a um periódico. 


\section{Personalizar}

Identificou-se um tipo de comportamento que não se encaixa nas categorias do modelo de Ellis, sendo necessária a criação de uma nova categoria denominada "personalizar". Ela descreve a tendência dos pesquisadores entrevistados, neste estudo, de personalizar, nos sistemas de recuperação de informações, os recursos de busca, adequando-os às suas necessidades ou do grupo ao qual estão vinculados. Exemplifica-se a categoria com a ação de adicionar todos os títulos dos sites que mais consulta aos "favoritos", ou realizar outras customizações no seu browser, como criar páginas específicas ou disponibilizar, pela Internet, links para os periódicos mais utilizados em seu campo de atuação, entre outros. Esse comportamento é destacado nos trechos que seguem:

[...] na página nossa do Departamento eu acabei criando um link para determinadas revistas, que são as que nós mais utilizamos [...] (Pesquisador D).

Este browser, o FireFox, é um avanço nesse sentido. Ele permite a você personalizar todas as barras. Quando você vai acessar, por exemplo... Esse, por exemplo, são notícias da Science... ele tem um link e você vai direto... esse sistema facilita muito. Eu o uso também para acessar outros sistemas (demonstra a pesquisa). O Pubmed você acessa aqui direto; esse browser está integrado, você pode acessar a página e fazer a pesquisa através dele, sem ir ao Pubmed (...). (Pesquisador D).

[...] eu tenho por hábito ter a própria página do periódico cadastrada no meu browser; já abre na página da Nature [...] (Pesquisador F).

A execução da categoria "personalizar" facilita as atividades relacionadas a outras categorias, como, por exemplo, "monitorar", pois todas as fontes estarão facilmente disponibilizadas, o que agilizará o acesso aos periódicos. Essa atividade também pode refletir a intenção dos pesquisadores de unir, em uma única ferramenta, todos os recursos e fontes de que precisa, facilitando seu acesso à informação.

Baseando-se na análise e identificação das categorias do modelo de David Ellis e na nova categoria verificada, é possível confirmar este modelo, acrescentando às categorias já definidas uma amplitude maior, gerada pelos novos recursos da comunicação e informação, principalmente pelos periódicos científicos eletrônicos.

\section{CONCLUSÕES}

O estudo realizado apresenta-se como uma verificação das práticas do comportamento de busca e uso de informação de um grupo específico.

Dentre as características levantadas, ressalta-se inicialmente aquelas inerentes à área da biotecnologia e biologia molecular, à qual os pesquisadores pertencem. Verifica-se o uso intenso dos recursos tecnológicos, de publicações científicas eletrônicas, de bases de dados, dominando os recursos de busca e acesso às informações. Vinculado a isso, verificou-se que, para a biotecnologia e biologia molecular, bem como para as áreas biomédicas em geral, existe uma quantidade expressiva de fontes de informação científica eletrônicas, com variados recursos de busca e recuperação de informações e de repositórios de textos na íntegra.

Em relação ao modelo de comportamento de busca e uso de informação de Ellis, ele foi confirmado, reconhecendo e ampliando as características originais.

Observou-se que os pesquisadores iniciam suas buscas em bases de dados on-line, informando os assuntos que desejam localizar. Após achar as referências, os artigos são obtidos por meio de links para seu conteúdo na íntegra, disponibilizados, principalmente, no Portal de Periódicos da Capes. Durante este processo, o pesquisador não precisa se deslocar de uma fonte para outra, como fazia anteriormente, pois agora, com a disponibilização das fontes de forma eletrônica e on-line com documentos na íntegra, a obtenção é imediata. Relata-se ainda que, para alguns pesquisadores, a busca, a localização e a obtenção dos artigos está unificada e é realizada somente por uma fonte de informação eletrônica.

Ainda, em relação aos estudos de comportamento, especificamente o modelo de Ellis, deve ser destacado que, devido à crescente utilização da tecnologia, a maioria das categorias tradicionais se alterara. Estas alterações resultaram na ampliação do seu alcance, como ocorreu com a categoria "monitorar", a qual se tornou mais efetiva com o uso de recursos tecnológicos, pois foram utilizadas ferramentas que permitem que as ações vinculadas a esse comportamento específico se dêem de modo mais simplificado e eficiente, como, por exemplo, mediante o uso de avisos automáticos enviados por e-mail.

Como sugestões, recomenda-se a realização de novos estudos sobre o processo de busca e uso de informação, preferencialmente com outras áreas do conhecimento, 
para permitir a comparação das características levantadas e possibilitar que se obtenha um conhecimento/ entendimento mais abrangente da comunicação científica em meio eletrônico.

Propõe-se, ainda, um estudo bibliométrico para verificar se a mudança de comportamento de busca e uso de informação se reflete nas citações dos trabalhos dos pesquisadores entrevistados, ou seja, para identificar, pela sua produção, o tipo e a origem dos materiais que estão sendo citados, se são, por exemplo, oriundos de periódicos científicos eletrônicos de acesso livre.

Artigo submetido em 13/03/2006 e aceito em 22/03/2007.

\section{REFERÊNCIAS}

COElHO, B. A. de S. et al. Estudos de usuário e comunicação científica: relações implícitas e explícitas. Ciência da Informação, Brasília, v. 18, n. 1, p. 62-73, jan./jun. 1989.

CHOO, C. W.; DETLOR, B.; TURNBULL, D. A behavioral model of information seeking on the web: preliminary results of study of how managers and IT specialists use the web. In: ASIS ANNUAL MEETING, 35., 1998, Medford. Electronic proceedings.... Medford: [s. n.], 1998. Disponível em: < http://choo.fis.utoronto.ca/fis/respub/asis98/ >. Acesso em: 27 out. 2004.

Information seeking on the web: an integrated model of browsing and searching. First Monday, Washington, DC, v. 5, n. 2, Feb. 2000. Disponível em: < http://firstmonday.org/issues/issues5_2/ choo/index.html>. Acesso em: 27 out. 2004.

DERVIN, B.; NILAN, M. Information needs and uses. Annual Review of Information Science and Technology, v. 21, p. 3-33, 1986. White Plains.

ELLIS, D. A. Behavioral approach to information retrieval system design. Journal of Documentation, London, v. 45, n. 3, p. 171-212, Sept. 1989a.

. A behavioral model for information retrieval system design. Journal of Information Science, Cambridge, n. 15, p. 237-247, 1989 b.
; COX, D.; HALL, K. A comparison of the information seeking patterns of researchers in the physical and social sciences. Journal of Documentation, London, v. 49, n. 4, p. 356-369, 1993.

FLICK, U. Uma introdução à pesquisa qualitativa. 2. ed. Porto Alegre: Bookman, 2004. 312 p.

GREFSHEIM, S.; FRANKLIN, J.; CUNNINGHAM, D. Biotechnology awareness study, part 1: where scientists get their information. Bulletin of the Medical Library Association, Chicago, v. 79, n. 1, p. 36-44, Jan. 1991.

KRIKELAS, J. Information seeking behavior: patterns of academic researchers. Drexel Library Quaterly, Philadelphia, v. 19, p. 5-20, 1983.

KUHLTHAU, Carol C. Inside de search process: information seeking from the user's perspective. Journal of the American Society for Information Science, Washington, DC, v. 42, n. 5, p. 361-371, 1991.

MARCHIONINI, Gary. Information seeking in electronic environments. Cambridge: Cambridge University Press, 1998. 224 p.

MEADOWS, J. A comunicação científica. Brasília: Briquet de Lemos, 1999.268 p.

MEHO, L.; TIBBO, H. R. Modeling the information-seeking behavior of social scientists: Ellis's study revisited. Journal of the American Society for Information Science and Technology, New York, v. 54, n. 6, p. 570-587, 2003.

STRAUSS, A.; CORBIN, J. Basics of qualitative research: grounded theory and procedures and techniques. Newbury Park: SAGE, 1990. $270 \mathrm{p}$.

TEIXEIRA, A. N.; BECKER, F. Novas possibilidades da pesquisa qualitativa via sistema CAQDAS. Sociologias, Porto Alegre, v. 3, n. 5, p. 94-114, jan./jun. 2001.

WILSON, T. D. Information behaviour: an interdisciplinary perspective. Information Processing $\mathscr{E}$ Management, Elmsford, v. 33, n. 4, p. 551-572, 1997.

. Models in information behaviour research. Journal of Documentation, London, v. 55, n. 4, p. 249-270, June 1999.

. On user studies and information need's. Journal of Documentation, London, v. 37, n. 1, p. 3-15, Mar. 1981.

ZIMAN, J. M. Conhecimento público. Belo Horizonte: Itatiaia, 1979. $164 \mathrm{p}$. 